\title{
EVERY WIJSMAN TOPOLOGY RELATIVE TO A POLISH SPACE IS POLISH
}

\author{
C. COSTANTINI
}

(Communicated by Franklin D. Tall)

\begin{abstract}
Generalizing a result of G. Beer and a result of E. Effros, we show that if $(X, d)$ is a separable and completely metrizable metric space, then the hyperspace of $X$ endowed with the Wijsman topology is separable and completely metrizable.
\end{abstract}

\section{INTRODUCTION}

In [Be], G. Beer proved that if $(X, d)$ is a separable complete metric space, then the Wijsman topology on the hyperspace of $X$ is Polish, i.e. separable and completely metrizable.

In the same paper, the author emphasizes the essential role played by Polish spaces in descriptive set theory and its applications to measurable multifunctions and probability theory. Thus, the problem of finding Polish topologies for the hyperspace of a Polish space may take on some importance for various mathematical fields.

With the article [Ef] of 1965, E. Effros already provided an effective result in this direction. He established that if $X$ is a Polish space, then considering any totally bounded compatible metric $\rho$ on $X$ (see for example [En], Theorem 4.3.5), the Hausdorff topology $H_{\rho}$ relative to this metric on the hyperspace of $X$ is Polish. Observe that, by Corollary 5.7 of [BLLN], for a totally bounded metric space $(X, \rho)$ the Hausdorff topology $H_{\rho}$ and the Wijsman topology $w_{\rho}$ agree. Therefore the article of Effros is, in fact, the first to endow the hyperspace of a Polish space with a (suitable) Polish Wijsman topology.

The aim of our paper is to prove that if $X$ is a Polish space, then for every compatible metric $d$ on $X$ the relative Wijsman topology $w_{d}$ on the collection of all closed (nonempty) subsets of $X$ is Polish. This result clearly generalizes both the preceding ones, but it cannot be automatically deduced from either of them, as, of course, equivalent metrics on a set $X$ may give rise to different Wijsman topologies on the hyperspace of $X$ (see, in particular, Theorems 5 and $5^{\prime}$ of [CLZ]). Let us note that, in a similar vein, A. Barbati proved that the Wijsman hyperspace of an analytic space $X$ is analytic, for any choice of a compatible metric $d$ on $X$ ([Ba1], Theorems III.2.i and II.8, or [Ba2]).

Received by the editors October 7, 1993.

1991 Mathematics Subject Classification. Primary 54B20; Secondary 54E50, 54D65.

$K e y$ words and phrases. Wijsman topology, Polish space, completely metrizable space, $G_{\delta}$-subset. 


\section{DEFINITIONS AND BASIC RESULTS}

Let $(X, d)$ be a metric space and $c_{0}(X)$ the collection of all closed nonemtpy subsets of $X$. Following [Be], section 3, we will define the Wijsman topology on $c_{0}(X)$ as the weak topology generated by $\{d(x, \cdot) \mid x \in X\}$, where each $d(x, \cdot)$ denotes the functional from $c_{0}(X)$ to $[0,+\infty)$ associating to every $A \in c_{0}(X)$ the nonnegative real number $d(x, A)$. As observed in the same section of [Be], having fixed any dense subset $D$ of $X$, the collection

$$
\begin{aligned}
& \left\{d(x, \cdot)^{-1}(\alpha, \beta) \mid x \in D, \alpha, \beta \text { rational, } \alpha<\beta\right\} \\
& \quad=\left\{\left\{A \in c_{0}(X) \mid \alpha<d(x, A)<\beta\right\} \mid x \in D, \alpha, \beta \text { rational, } \alpha<\beta\right\}
\end{aligned}
$$

serves as our canonical subbase for $w_{d}$.

For every $x \in X$ and every $\alpha, \beta \in \mathbf{R}$ with $\alpha<\beta$, we will define

$$
\mathscr{A}_{x, \alpha, \beta}=\left\{A \in c_{0}(X) \mid \alpha<d(x, A)<\beta\right\}
$$

thus each $\mathscr{A}_{x, \alpha, \beta}$ is an open subset of $\left(c_{0}(X), w_{d}\right)$ and

$$
\Delta=\left\{\mathscr{A}_{x, \alpha, \beta} \mid x \in D, \alpha, \beta \text { rational, } \alpha<\beta\right\}
$$

is our canonical subbase for $\left(c_{0}(X), w_{d}\right)$.

Moreover, for every $x \in X$ and $\varepsilon>0$ we define $\mathscr{A}_{x, \varepsilon}^{-}=\left\{A \in c_{0}(X) \mid d(x, A)\right.$ $<\varepsilon\}$ and $\mathscr{A}_{x, \varepsilon}^{+}=\left\{A \in c_{0}(X) \mid d(x, A)>\varepsilon\right\}$, which are, as well, open subsets of $\left(c_{0}(X), w_{d}\right)$.

Now, let $(\tilde{X}, \tilde{d})$ be the completion of $(X, d)$. As $D$ is dense in $X$, it is dense in $\tilde{X}$ too, and the collection

$$
\widetilde{\Delta}=\left\{\widetilde{\mathscr{A}}_{x, \alpha, \beta} \mid x \in D, \alpha, \beta \text { rational, } \alpha<\beta\right\}
$$

-where $\widetilde{\mathscr{A}_{x^{\prime}}, \alpha^{\prime}, \beta^{\prime}}=\left\{A \in c_{0}(\tilde{X}) \mid \alpha^{\prime}<\tilde{d}\left(x^{\prime}, A\right)<\beta^{\prime}\right\}$ for every $x^{\prime} \in X$ and $\alpha^{\prime}, \beta^{\prime} \in \mathbf{R}$ with $\alpha^{\prime}<\beta^{\prime}$-is our canonical subbase for $c_{0}(\tilde{X})$.

Similarly, we will define for every $x \in \tilde{X}$ and $\varepsilon>0: \widetilde{\mathscr{A}_{x, \varepsilon}^{-}}=\left\{A \in c_{0}(\tilde{X}) \mid\right.$ $\tilde{d}(x, A)<\varepsilon\}$ and $\widetilde{\mathscr{A}_{x, \varepsilon}^{+}}=\left\{A \in c_{0}(\tilde{X}) \mid \tilde{d}(x, A)>\varepsilon\right\}$ which are, in turn, open subsets of $\left(c_{0}(\tilde{X}), w_{\tilde{d}}\right)$.

Finally, define the map $\tilde{j}: c_{0}(X) \rightarrow c_{0}(\tilde{X})$ as $\tilde{\jmath}(A)=C l_{\widetilde{X}}(A)$. We will show that $\tilde{j}$ is a topological embedding of $\left(c_{0}(X), w_{d}\right)$ into $\left(c_{0}(\tilde{X}), w_{\tilde{d}}\right)$. [Note that in the paper of Effros it is proved, in an analogous situation, that $\tilde{j}$ is a topological embedding of $\left(c_{0}(X), H_{d}\right)$ into $\left(c_{0}(\tilde{X}), H_{\tilde{d}}\right)$.]

To prove injectivity, take $A, A^{\prime} \in c_{0}(X)$ with $A \neq A^{\prime}$, and suppose for instance that there exists $x \in A \backslash A^{\prime}$ : as $A^{\prime}$ is closed in $X$, we see that $x \notin$ $C l_{\widetilde{X}}\left(A^{\prime}\right)=\tilde{\jmath}\left(A^{\prime}\right)$, and hence $\tilde{\jmath}(A) \neq \tilde{\jmath}\left(A^{\prime}\right)$.

Concerning continuity, consider first that for every $y \in \tilde{X}$ and every $M \subseteq \tilde{X}$ we have $\tilde{d}(y, M)=\tilde{d}\left(y, C l_{\tilde{X}}(M)\right)$; thus it is easy to see that for every $x \in D$ and every pair $\alpha, \beta$ of rational numbers with $\alpha<\beta$ we have $\tilde{j}^{-1}\left(\widetilde{\mathscr{A}}_{x, \alpha, \beta}\right)=$ $\mathscr{A}_{x, \alpha, \beta}$.

Finally, to prove the open character of $\tilde{j}$ onto its range, observe that, by injectivity, it will suffice to show that the image of every element of $\Delta$ is open in $\tilde{j}\left(c_{0}(X)\right)$. In fact, for every $\mathscr{A}_{x, \alpha, \beta} \in \Delta$ we have $\tilde{\jmath}\left(\mathscr{A}_{x, \alpha, \beta}\right)=\widetilde{\mathscr{A}}_{x, \alpha, \beta} \cap$ $\tilde{j}\left(c_{0}(X)\right)$. 


\section{THE MAIN THEOREM}

Let $(X, d)$ be a separable completely metrizable space, and $D=\left\{x_{m} \mid m \in\right.$ $\left.\mathbf{Z}^{+}\right\}$be a countable dense subset of $X$. Denoting, as above, by $(\tilde{X}, \tilde{d})$ the completion of $(X, d)$, we know that $X$ is a $G_{\delta}$-subset of $\widetilde{X}$ (see [En], Theorem 4.3.24). Thus there exists a sequence $\left(\widetilde{F}_{n}\right)_{n \in \mathbf{Z}^{+}}$of closed subsets of $\widetilde{X}$ such that $\tilde{X} \backslash X=\bigcup_{n \in \mathbf{Z}^{+}} \widetilde{F}_{n}$.

We wish to prove that $\left(c_{0}(X), w_{d}\right)$ is a Polish space. Since we found that the map $\tilde{j}:\left(c_{0}(X), w_{d}\right) \rightarrow\left(c_{0}(\tilde{X}), w_{\tilde{d}}\right)$ is a topological embedding, and $\left(c_{0}(\tilde{X}), w_{\tilde{d}}\right)$ is a Polish space by [Be], we only have to show that $\tilde{j}\left(c_{0}(X)\right)$ is a $G_{\delta}$-subset of $\left(c_{0}(\tilde{X}), w_{\tilde{d}}\right)$.

For every $x \in \widetilde{X}$ and $\varepsilon>0$, put $S_{\tilde{d}}(x, \varepsilon)=\{y \in \tilde{X} \mid \tilde{d}(x, y)<\varepsilon\}$ and $\bar{S}_{\tilde{d}}(x, \varepsilon)=\{y \in \tilde{X} \mid \tilde{d}(x, y) \leq \varepsilon\}$. For every open subset $\Omega$ of $\tilde{X}$, put:

$$
\Omega^{-}=\left\{A \in c_{0}(\tilde{X}) \mid A \cap \Omega \neq \varnothing\right\} .
$$

It is a well-known fact that for every open subset $\Omega$ of $\tilde{X}, \Omega^{-}$is $w_{\tilde{d}^{-}}$open. Indeed, for every $x \in \Omega$ fix $\varepsilon_{x}>0$ such that $S_{\tilde{d}}\left(x, \varepsilon_{x}\right) \subseteq \Omega$; then we have $\Omega^{-}=\bigcup_{x \in \Omega^{-}} \widetilde{\mathscr{A}_{x, \varepsilon_{x}}^{-}}$.

Finally, for every triple $(m, n, p)$ of positive integers, put

$$
\mathscr{G}_{m, n, p}=\left(S_{\tilde{d}}\left(x_{m}, \frac{1}{p}\right) \backslash \widetilde{F}_{n}\right)^{-} \cup \widetilde{\mathscr{A}}_{x_{m}, \frac{1}{p+1}} .
$$

It follows from the preceding remarks that each $\mathscr{G}_{m, n, p}$ is $w_{\tilde{d}}$-open. We claim that

$$
\tilde{\jmath}\left(c_{0}(X)\right)=\bigcap_{m, n, p \in \mathbf{Z}^{+}} \mathscr{G}_{m, n, p}
$$

which would complete the proof.

Concerning the inclusion " $\subseteq$ ", take $A \in c_{0}(X)$ and let $m, n, p$ be arbitrary elements of $\mathbf{Z}^{+}$: if $\tilde{d}\left(x_{m}, \tilde{j}(A)\right)>1 /(p+1)$, then $\tilde{j}(A) \in \mathscr{S}_{m, n, p}$. Suppose $\tilde{d}\left(x_{m}, \tilde{\jmath}(A)\right) \leq 1 /(p+1)<1 / p$; then $S_{\tilde{d}}\left(x_{m}, 1 / p\right) \cap \tilde{\jmath}(A) \neq \varnothing$ and there exists $\bar{y} \in S_{\tilde{d}}\left(x_{m}, 1 / p\right) \cap \tilde{j}(A)$.

As $\tilde{\jmath}(A)=C l_{\widetilde{X}}(A)$ and $S_{\tilde{d}}\left(x_{m}, 1 / p\right)$ is an open neighbourhood of $\bar{y}$, the set $A \cap S_{\tilde{d}}\left(x_{m}, 1 / p\right)$ is nonempty and must contain a point $z$. Then $z \notin \widetilde{F}_{n}$ (because $\widetilde{F}_{n} \cap A \subseteq \widetilde{F}_{n} \cap X=\varnothing$ ), and hence

$$
\left(S_{\tilde{d}}\left(x_{m}, 1 / p\right) \backslash \widetilde{F}_{n}\right) \cap \tilde{\jmath}(A) \neq \varnothing,
$$

i.e. $\tilde{j}(A) \in\left(S_{\tilde{d}}\left(x_{m}, 1 / p\right) \backslash \widetilde{F}_{n}\right)^{-}$.

Before establishing the opposite inclusion, it is convenient to state a result which will be needed in the proof.

Lemma. Let $(\tilde{X}, \tilde{d})$ be a complete metric space and $\left(\widetilde{F}_{n}\right)_{n \in \mathbf{Z}^{+}}$a sequence of closed subsets of $\widetilde{X}$; put $\widetilde{F}=\bigcup_{n \in \mathbf{Z}^{+}} \widetilde{F}_{n}$. Suppose $\widetilde{D}$ is a closed subset of $\widetilde{X}$ contained in $\widetilde{F}$. Then:

$$
\forall x \in \widetilde{D}: \forall \varepsilon>0: \exists y \in S_{\tilde{d}}(x, \varepsilon) \cap \widetilde{D}: \exists \delta>0: \exists n \in \mathbf{Z}^{+}: S_{\tilde{d}}(y, \delta) \cap \widetilde{D} \subseteq \widetilde{F}_{n} .
$$


Proof. The set $S_{\tilde{d}}(x, \varepsilon) \cap \widetilde{D}$ is a Baire space, as a $G_{\delta}$-subset of $\widetilde{X}$. From $S_{\tilde{d}}(x, \varepsilon) \cap \widetilde{D}=\bigcup_{n=1}^{\infty}\left(\left(S_{\tilde{d}}(x, \varepsilon) \cap \widetilde{D}\right) \cap \widetilde{F}_{n}\right)$, we obtain that there exist $n \in \mathbf{Z}^{+}$, $y \in\left(S_{\tilde{d}}(x, \varepsilon) \cap \widetilde{D}\right) \cap \widetilde{F}_{n}$ and $\delta^{\prime}>0$ such that $S_{\tilde{d}}\left(y, \delta^{\prime}\right) \cap\left(S_{\tilde{d}}(x, \varepsilon) \cap \widetilde{D}\right) \subseteq$ $\left(S_{\tilde{d}}(x, \varepsilon) \cap \widetilde{D}\right) \cap \widetilde{F}_{n} \subseteq \widetilde{F}_{n}$. Putting $\delta=\min \left\{\delta^{\prime}, \varepsilon-\tilde{d}(x, y)\right\}$ gives the result.

We can now complete the proof of $(\nabla)$. We have to show that if $\tilde{C} \in$ $c_{0}(\tilde{X}) \backslash \tilde{j}\left(c_{0}(X)\right)$, then there exist $m, n, p \in \mathbf{Z}^{+}$such that $\widetilde{C} \notin \mathscr{G}_{m, n, p}$.

Indeed, if $\widetilde{C} \notin \tilde{j}\left(c_{0}(X)\right)$, then $\widetilde{C} \neq C l_{\widetilde{X}}(\tilde{C} \cap X)$ and hence we can find $\bar{x} \in$ $\tilde{C} \backslash C l_{\tilde{\tilde{X}}}(\tilde{C} \cap X)$; choose $r>0$ such that $S_{\tilde{d}}(\bar{x}, r)$ does not meet $\tilde{C} \cap X$, and put $\widetilde{D}=\tilde{C} \cap \bar{S}_{\tilde{d}}(\bar{x},(1 / 2) r)$. It follows that $\widetilde{D}$ is closed in $\widetilde{X}$ and that $\widetilde{D} \cap X=\varnothing$, i.e. $\tilde{D} \subseteq \widetilde{X} \backslash X=\bigcup_{n \in \mathbf{Z}^{+}} \widetilde{F}_{n}$. Applying the lemma [with $\widetilde{F}=\tilde{X} \backslash X, x=\bar{X}$ and $\varepsilon=(1 / 2) r]$ we see that there exist $\bar{y} \in \widetilde{D}, \delta>0$ and $\bar{n} \in \mathbf{Z}^{+}$such that $\tilde{d}(\bar{x}, \bar{y})<(1 / 2) r$ and

$$
S_{\tilde{d}}(\bar{y}, \delta) \cap \widetilde{D} \subseteq \widetilde{F}_{\bar{n}}
$$

moreover, $\delta$ can be so chosen that

$$
\delta \leq \frac{1}{2} r-\tilde{d}(\bar{x}, \bar{y}) .
$$
$\delta-\sigma$.

Take $\sigma$ with $0<\sigma<\delta$ and $p \in \mathbf{Z}^{+}$such that $\sigma<1 /(p+1)$ and $1 / p<$

Finally, let $\bar{m} \in \mathbf{Z}^{+}$be such that

$$
\tilde{d}\left(x_{\bar{m}}, \bar{y}\right)<\sigma .
$$

We will prove that $\widetilde{C} \notin \mathscr{G}_{\bar{m}, \bar{n}, p}$.

First, it is clear that, since $\bar{y} \in \widetilde{D} \subseteq \tilde{C}$, we obtain $\tilde{d}\left(x_{\bar{m}}, \tilde{C}\right) \leq \tilde{d}\left(x_{\bar{m}}, \bar{y}\right)<$ $\sigma<1 /(p+1)$ and hence $\widetilde{C} \notin \widetilde{\mathscr{A}}_{x_{\bar{m}}^{+}, 1 /(p+1)}$. To show that

$$
\tilde{C} \notin\left(S_{\dot{d}}\left(x_{\bar{m}}, 1 / p\right) \backslash \widetilde{F}_{\bar{n}}\right)^{-},
$$

i.e. that $\widetilde{C} \cap S_{\tilde{d}}\left(x_{\bar{m}}, 1 / p\right) \subseteq \widetilde{F}_{\bar{n}}$, it will suffice [by $\left.(\star)\right]$ to establish that

$$
\widetilde{C} \cap S_{\tilde{d}}\left(x_{\bar{m}}, 1 / p\right) \subseteq \widetilde{D} \cap S_{\dot{d}}(\bar{y}, \delta)=\tilde{C} \cap \bar{S}_{\bar{d}}(\bar{x},(1 / 2) r) \cap S_{\tilde{d}}(\bar{y}, \delta) .
$$

Note that the last set is equal to $\tilde{C} \cap S_{\tilde{d}}(\bar{y}, \delta)$, because

$$
S_{\bar{d}}(\bar{y}, \delta) \subseteq S_{\bar{d}}(\bar{x},(1 / 2) r) \subseteq \bar{S}_{\bar{d}}(\bar{x},(1 / 2) r) ;
$$

indeed, if $z$ is such that $\tilde{d}(\bar{y}, z)<\delta$, we deduce by (\#) that $\tilde{d}(\bar{x}, z) \leq$ $\tilde{d}(\bar{x}, \bar{y})+\tilde{d}(\bar{y}, z)<\tilde{d}(\bar{x}, \bar{y})+(1 / 2) r-\tilde{d}(\bar{x}, \bar{y})=(1 / 2) r$. Thus we will complete the proof by showing that $S_{\dot{d}}\left(x_{\bar{m}}, 1 / p\right) \subseteq S_{\dot{d}}(\bar{y}, \delta)$.

Let $z \in \widetilde{X}$ be such that $\tilde{d}\left(x_{\bar{m}}, z\right)<1 / p$ : as $1 / p<\delta-\sigma$, we conclude by $(\diamond)$ that $\tilde{d}(\bar{y}, z) \leq \tilde{d}\left(\bar{y}, x_{\bar{m}}\right)+\tilde{d}\left(x_{\bar{m}}, z\right)<\sigma+\delta-\sigma=\delta$.

\section{FinAL OBSERVATIONS}

As remarked in $[\mathrm{Be}]$, p. 1129 , for every metric space $(X, d)$ the complete metrizability of $X$ is a necessary condition for the Wijsman topology $\left(c_{0}(X), w_{d}\right)$ to be completely metrizable [since $X$ can be embedded in 
$\left(c_{0}(X), w_{d}\right)$ as a closed subset]. As it is well known that separability of $(X, d)$ is equivalent to metrizability of $\left(c_{0}(X), w_{d}\right)$ (see [LL], Theorem 3.5), we can state the result of the preceding section in the following form:

Theorem 1. For every metric space $(X, d)$, the space $\left(c_{0}(X), w_{d}\right)$ is Polish if and only if $X$ is Polish.

In the literature, there exists a classical way to extend the Wijsman topology to the whole collection $c(X)$ of closed subsets of a metric space $(X, d)$ (see for instance [LL], section 1); following such a definition, a fundamental system of neighbourhoods for the empty set is given by all collections of the form $\mathscr{A}_{x, \varepsilon}^{+}$, with $x \in X$ and $\varepsilon>0$ (where we put $d(x, \varnothing)=+\infty$ for every $x \in X$ ). This extension is still $T_{1}$ (and completely regular); in particular, $c_{0}(X)$ is open in $c(X)$. Moreover, separability of $X$ still implies separability and metrizability of $\left(c(X), w_{d}\right)$ ([FLL], Theorem 4.2). These results allow us to extend Theorem 1 to $c(X)$.

Theorem 2. For every metric space $(X, d)$, the space $\left(c(X), w_{d}\right)$ is Polish if and only if $X$ is Polish.

Proof. If $\left(c(X), w_{d}\right)$ is Polish, then the open subspace $\left(c_{0}(X), w_{d}\right)$ is Polish and hence $X$ is Polish (Theorem 1).

Conversely, if $X$ is Polish, then $\left(c_{0}(X), w_{d}\right)$ is Polish. Consider a metrizable compactification $\mathscr{K}$ of $\left(c(X), w_{d}\right)$, which must exist as $\left(c(X), w_{d}\right)$ is separable and metrizable. Then $\left(c_{0}(X), w_{d}\right)$ is a $G_{\delta}$-subset of $\mathscr{K}$ (see [En], Theorem 4.3.24). Thus $c(X)=c_{0}(X) \cup\{\varnothing\}$ is in turn a $G_{\delta}$-subset of $\mathscr{K}$, and hence is completely metrizable.

Note that the last argument of this proof is similar to the proof of Theorem 4.4 in [Be]. Nevertheless, local compactness is not necessary for a separable metrizable space to have a metrizable compactification.

\section{ACKNOWLEDGMENT}

To conclude this paper, the author wishes to thank C. Hess for continuously encouraging his work, and S. Levi for simplifying the original proof of the main result.

\section{REFERENCES}

[Ba1] A. Barbati, Strutture boreliane sugli iperspazi, Tesi di Laurea, Università di Milano, 1993.

[Ba2] - The hyperspace of an analytic metrizable space is analytic, Proc. 11 th International Conference on Topology (Trieste, September 1993); Rend. Ist. Mat. Trieste 25 (1993).

[Be] G. Beer, A Polish topology for the closed subsets of a Polish space, Proc. Amer. Math. Soc. 113 (1991), 1123-1133.

[BLLN] G. Beer, A. Lechicki, S. Levi, and S. Naimpally, Distance functionals and suprema of hyperspace topologies, Ann. Mat. Pura Appl. (4) 162 (1992), 367-381.

[CLZ] C. Costantini, S. Levi, and J. Zieminska, Metrics that generate the same hyperspace convergence, Set-Valued Analysis 1 (1993), 141-157.

[Ef] E. Effros, Convergence of closed subsets in a topological space, Proc. Amer. Math. Soc. 16 (1965), 929-931.

[En] R. Engelking, General topology, revised and completed edition, Heldermann Verlag, Berlin, 1989. 
[FLL] S. Francaviglia, A. Lechicki, and S. Levi, Quasi-uniformization of hyperspaces and convergence of nets of semicontinuous multifunctions, J. Math. Anal. Appl. 112 (1985), 347-370.

[LL] A. Lechicki and S. Levi, Wijsman convergence in the hyperspace of a metric space, Boll. Un. Mat. Ital. (7) B.1 (1987), 439-451.

Dipartimento di Matematica, Universita di Milano, Via Saldini 50, 20133 Milano, ITALY 